\title{
miR-146b-5p promotes invasion and metastasis contributing to chemoresistance in osteosarcoma by targeting zinc and ring finger 3
}

\author{
ENJIE XU* , JIANQUAN ZHAO ${ }^{*}$, JUN MA, CE WANG, CHENGLIN ZHANG, \\ HENG JIANG, JIANYANG CHENG, RUI GAO and XUHUI ZHOU
}

Department of Orthopedic Surgery, Changzheng Hospital, Second Military Medical University, Shanghai 200003, P.R. China

Received June 8, 2015; Accepted July 10, 2015

DOI: $10.3892 /$ or.2015.4393

\begin{abstract}
Osteosarcoma is the most common human primary malignant bone tumor and recurrences are common due to the development of chemoresistance. However, the underlying molecular mechanism for chemoresistance remains unclear. Recent studies demonstrated that miR-146b-5p, an important regulator in tumorigenesis, was involved in chemoresistance in thyroid cancer, lymphoma. Thus, to confirm the role of miR-146b-5p in osteosarcoma, the study was divided into three steps: first, miR-146b-5p in paired samples were assessed using a quantitative real-time PCR (qRT-PCR) assay from osteosarcoma patients. Second, to confirm the role of miR-146b-5p, we applied lentivirus system to overexpression and knockdown of miR-146b-5p, respectively, in MG-63 osteosarcoma cell line. Third, luciferase assays were performed to determine whether Wnt/ $\beta$-catenin pathway participated in the role of miR-146b-5p on chemoresistance. As a result, miR-146b-5p was highly expressed in human osteosarcoma tissues and an elevated expression of miR-146b-5p was observed in human osteosarcoma tissues after chemotherapy. Furthermore, it was shown that miR-146b-5p overexpression promoted migration and invasiveness. miR-146b-5p overexpression also increased resistance to chemotherapy. Moreover, knockdown of miR$146 b-5 p$ substantially inhibited migration and invasion of osteosarcoma cells as well as rendered them significantly more sensitive to chemotherapy. Results of western blot assay indicated that miR-146b-5p increased MMP-16 protein expression and showed a decrease of ZNRF3 protein. Whereas, IWR-1-
\end{abstract}

Correspondence to: Dr Xuhui Zhou or Dr Rui Gao, Department of Orthopedic Surgery, Changzheng Hospital, Second Military Medical University, 415 Fengyang Road, Shanghai 200003, P.R. China

E-mail: xuhuizhou66@163.com

E-mail: gaoruispine@126.com

*Contributed equally

Key words: miR-146b-5p, invasion, metastasis, chemoresistance, ZNRF3 endo, an inhibitor of $\mathrm{Wnt} / \beta$-catenin, suppressed the decrease in apoptosis of osteosarcoma cells caused by miR-146b-5p overexpression. These results indicated that miR-146b-5p promoted proliferation, migration and invasiveness. It also increased resistance to chemotherapy through the regulation of ZNRF3, and suggested novel potential therapeutic targets for the treatment of osteosarcoma.

\section{Introduction}

Osteosarcoma is the most common malignant bone tumor (1). The survival outcomes remain unsatisfactory since recurrences are common due to the development of invasion, distant metastasis and chemoresistance. Chemoresistance, both intrinsic and acquired, is a main cause of recurrence or failure of current treatment (2). Up to date, several factors contributed to the development of chemoresistance including genetic alterations (3), altered drug accumulation (4), drug-target amplification (5) and autophagy (6). However, the underlying molecular mechanism for chemoresistance remains unclear.

MicroRNAs (miRNAs) are small (22-nucleotide) noncoding single stranded RNAs that regulate gene expression by binding to the 3'-untranslated region (3'UTR) of their target mRNAs, modulating mRNA stability and protein expression at the post-transcriptional level. The aberrant expression of miRNAs has been implicated in the mechanism of chemoresistance (7-9) and several miRNAs have been identified in osteosarcoma tissues and osteosarcoma cell lines $(10,11)$.

miR-146b-5p was originally verified as involved in inflammatory bowel disease and was later shown upregulated in IL-10 deficient mice (12). Subsequently, it was found that miR$146 \mathrm{~b}-5 \mathrm{p}$ alleviated intestinal injury in mouse colitis via the activation of NF- $\kappa$ B. Recently, miR-146b-5p has been reported to be involved in solid tumors, including prostate, pancreatic cancer, malignant gliomas and glioblastoma. Moreover, two studies demonstrated that its involvement in chemoresistance in thyroid cancer (13) and lymphoma (14). However, the role of miR-146b-5p in osteosarcoma chemoresistance remains elusive.

Activation of $\mathrm{Wnt} / \beta$-catenin is a crucial step in the process of tumorigenesis and chemoresistance (15). Zinc and ring finger 3 (ZNRF3), an cell-surface transmembrane 
E3 ubiquitin ligase, negatively regulates $W n t / \beta$-catenin signalling by promoting the turnover of frizzled and LRP6. Originally, ZNRF3 was found to regulate the growth and survival of embryos stem cells and two previous studies demonstrated that ZNRF3-deficient embryos died around birth $(16,17)$. Moreover, ZNRF3 is also involved in the process of tumorigenesis, migration and invasiveness. Deng et al (13) reported that ZNRF3 suppressed the effect of miR-146b-5p on migration, invasiveness and proliferation of papillary thyroid cancer cells. ZNRF3 has also been confirmed to be linked to pancreatic ductal adenocarcinoma and mucinous ovarian tumors $(18,19)$.

To date, however, the exact role of miR-146b in osteosarcoma is not well understood. In the present study, we investigated the expression of miR-146b-5p in osteosarcoma tissues. We demonstrated osteosarcoma samples frequently featured highly expressed miR-146b-5p comparing to the normal tissue counterparts. An elevated expression of miR-146b-5p was observed in human osteosarcoma tissues after chemotherapy. Further study showed that overexpression of miR-146b-5p promoted invasion, metastasis and chemoresistance of osteosarcoma cells. In addition, ZNRF3 was identified as the target gene of miR-146b-5p in osteosarcoma. All these results indicate miR-146b-5p and its downstream target gene ZNRF3 can be used to treat osteosarcoma chemoresistance in the future.

\section{Materials and methods}

Tissue samples and papillary carcinoma cell lines. All research involving human tissue samples was approved by the Ethics Review Committee of Second Military Medical University Shanghai and written informed consent was obtained from all participating patients. Tumor specimens and the adjacent non-cancer tissues (ANCT) were obtained from 35 osteosarcoma patients who underwent surgery in Shanghai Changzheng Hospital between January 2010 and January 2013. Among them, 13 patients had received chemotherapy at the time of the operation. Ten patients were confirmed to have metastasis.

Cell culture. Human osteosarcoma hFOB1.19, MG-63 and U-2 OS cell lines were purchased from ATCC and reserved in our laboratory as previously described (20). Human MG-63 and U-2 OS cell lines were maintained in high glucose DMEM medium supplemented with $10 \%$ fetal bovine serum (FBS; HyClone), $100 \mathrm{U} / \mathrm{ml}$ penicillin, and $100 \mu \mathrm{g} / \mathrm{ml}$ streptomycin, and were cultured in an incubator maintained at $5 \% \mathrm{CO}_{2}$ and $37^{\circ} \mathrm{C}$.

Quantitative real-time RT-PCR. Total RNA containing miRNA was extracted from surgical specimens or cell lines with TRIZol reagent (Invitrogen, Carlsbad, CA, USA) according to the manufacturer's instructions. For miRNA expression analysis, the miRNA was transcribed into cDNA using a TaqMan MicroRNA Reverse Transcription kit (Applied Biosystems) and RT primers provided with the miR-146b-5p TaqMan miRNA assay (Applied Biosystems) according to the manufacturer's instructions. miR-146b-5p and U6 small nuclear 2 (U6) expression was detected from the cDNA product, using TaqMan miRNA sequence-specific probes using SYBR Premix Ex Taq (Takara, Shiga, Japan) and ABI Prism 7500 sequence detection system (Applied Biosystems). U6 was used as a control. The fold-change in gene expression was calculated by the $2^{-\Delta \Delta \mathrm{CT}}$ method.

Overexpression and knockdown of miR-146b-5p. Recombinant lentiviruses containing overexpression of miR-146b-5p, or knocked down miR-146b-5p and miRNA control were purchased from Shanghai GeneChem Co., Ltd. (Shanghai, China). To generate the stable cell line, human MG-63 osteosarcoma cells were transfected with lentiviruses containing overexpression of miR-146b-5p (OE), knocked down miR-146b-5p or a blank lentivirus expression vectors (blank). After incubation for $72 \mathrm{~h}$, the supernatant containing lentivirus was removed and replaced with complete culture medium. Infection efficiency was confirmed by RT-PCR $96 \mathrm{~h}$ after transfection and the cells were selected with $2 \mu \mathrm{g} / \mathrm{ml}$ to establish stable cell lines.

miRNA target prediction. Candidate targets of miR-146b-5p and ZNRF3 were predicted by miRBase (http://www.mirbase. org/) and TargetScan (http://www.Targetscan.org/).

Luciferase reporter assay. For validation of ZNRF3 as a target gene of miR-146b-5p in osteosarcoma cells, luciferase assay was performed as previously described (21). Briefly, the target sequence was amplified by PCR using the following primers: forward, 5'-ACTAGTGTATAGCAGCACATTTCATTT-3' and the reverse primer 5'-AAGCTTCAGGGTCTTGTGTTAG TC-3' and cloned into pGL3-control vector through $K p n I$ and $X h o I$ sites. Mimic was synthesized according to the sequence of miR-146b-5p. A ZNRF3 3'-UTR segment (516 bp) containing the predicted miR-146b-5p binding site was cloned into the pGL3-control vector (Promega Corp., Madison, WI, USA) downstream of the firefly luciferase gene, after which the 3'-UTR luciferase reporter was obtained. The miR-146b-5p mimic and miR-146b-5p inhibitor used in the present study were synthesized and provided by the GenePharma.

Western blot analysis. Protein extracts from cells were prepared through a modified lysis buffer and then performed as previously described (20). The immunoreactive bands were visualized using ECL-PLUS/kit. The relative protein level was normalized to $\beta$-actin concentration. Antibodies against matrix metalloproteinase-2 (MMP-2), MMP-9, MMP-16, cleaved caspase-3, caspase-3, cleaved PARP PARP, ZNRF3 and $\beta$-actin were purchased from Cell Signaling Technology, Inc. (Danvers, MA, USA).

Cell proliferation assay. Cell growth was determined by the MTT assay according to the manufacturer's instructions. Briefly, cells in OE, KD and control groups were incubated in 96-well plates at a density of $1 \times 10^{4}$ cells/well. Cells were treated with $10 \mu \mathrm{l}$ MTT reagents at $0,1,2,3,4,5,6$ and 7 days and then measured at $450 \mathrm{~nm}$ with enzyme immunoassay analyzer (Bio-Rad Laboratories, Hercules, CA, USA).

Migration and invasion assays. The invasive potential of cells was measured in $6.5 \mathrm{~mm}$ Transwell with $8.0 \mathrm{~mm}$ pore 

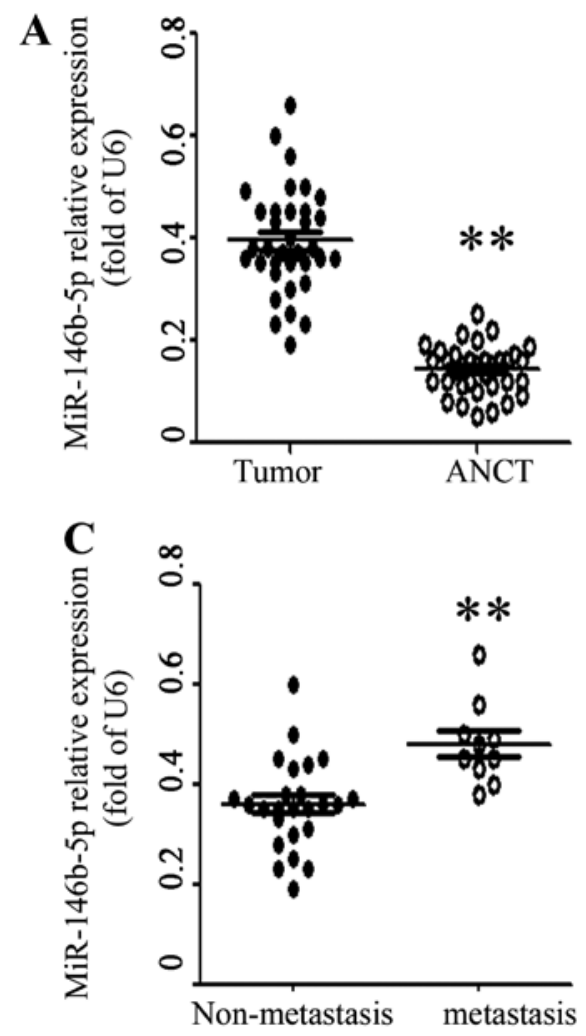

B

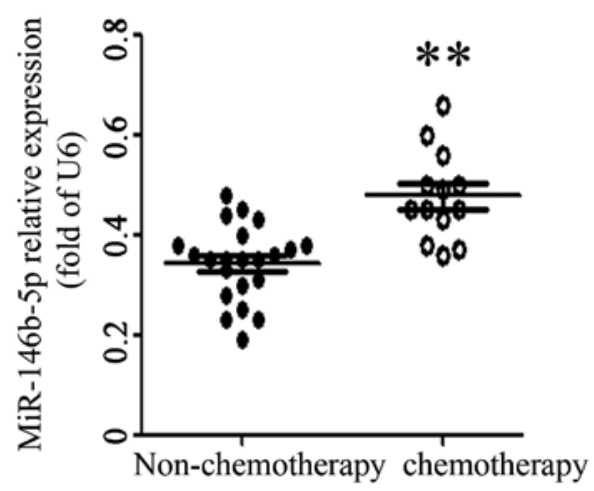

D

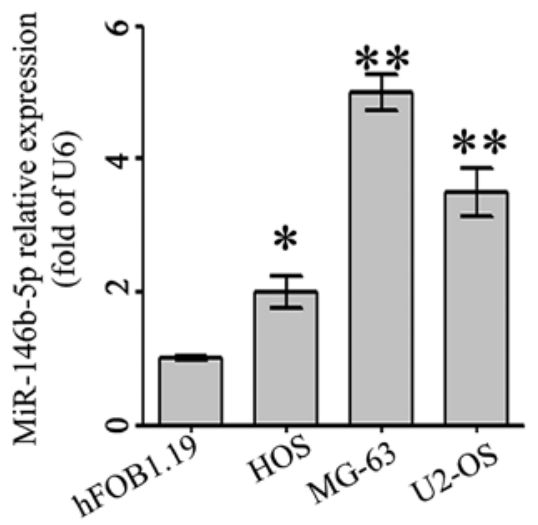

Figure 1. The expression levels of miR-146b-5p in osteosarcoma tissues and cell lines, as determined by qRT-PCR. Comparing differences in the expression levels of miR-146b-5p between (A) tumor tissues and ANCT; (B) non-chemotherapy group and chemotherapy group; (C) osteosarcoma tissues arising from metastasis and non-metastasis groups, and (D) HOS, MG-63, U2-OS osteosarcoma cell lines and the hFOB1.19 osteoblast cell line. * $\mathrm{P}<0.05$.

polycarbonate membrane insert (Corning, NY, USA). The filter of the top chamber was matrigel-coated with $50 \mu \mathrm{l}$ of diluted matrigel and the lower chamber was filled with $500 \mu \mathrm{l}$ of DMEM containing 10\% FBS. Cells were resuspended in migration medium (serum-free medium) and added into each top chamber. After the cells were incubated for $16 \mathrm{~h}$, the noninvading cells on the upper surface were removed by using a cotton swab. The invasive cells on the lower surface of the membrane insert were fixed with $4 \%$ paraformaldehyde for $30 \mathrm{~min}$, and then stained with hematoxylin-eosin. The number of cells on the lower surface, which had invaded through the membrane were counted. The procedure for Transwell migration assays were the same as the Transwell invasion assay except that the filter of top chamber was not coated with Matrigel. The number of cells were counted in five random fields of each chamber under the microscope.

Wound healing assay. When MG-63 cells in OE, KD and control groups were grown to confluence, a scratch was made with a micropipette tip. Following incubation for 24 and $48 \mathrm{~h}$, the images were captured. The migration potential was estimated by counting the cells that migrated from the wound edge. The cell migration rate was obtained by counting three fields per area and presented as the average of six independent evaluations.

Measurement of apoptosis by flow cytometry. The incidence of apoptosis after chemotherapy was assessed by using the
Annexin V-FITC /PI apoptosis detection kit (BD Pharmingen, San Diego, CA, USA) according to our previous method (20). Apoptotic cells, including those staining positive for Annexin V-FITC and negative for PI and those that were double positive, were counted as a percentage of the total cell count.

Statistical analysis. SPSS 13.0 was used for the statistical analysis. One-way analysis of variance (ANOVA) was used to analyze the differences between groups. The LSD method of multiple comparisons was employed when the probability for ANOVA was statistically significant. Statistical significance was $\mathrm{P}<0.05$.

\section{Results}

miR-146b-5p is upregulated in human osteosarcoma tissues after chemotherapy and correlates with multiple clinical features. First, we tested the expression of miR-146b-5p by qRT-PCR and normalized against an endogenous control (U6 RNA) in 35 pairs of tumor tissues and matched adjacent non-tumor tissues (ANCT) from osteosarcoma patients who received tumor resection. The expression level of miR-146b-5p in osteosarcoma tissues was significantly higher than that in matched ANCT ( $\mathrm{P}<0.05$; Fig. 1A). Next, osteosarcoma tissues sample were divided into patients that received chemotherapy (chemotherapy group) and those that did not (non-chemotherapy group). 
A

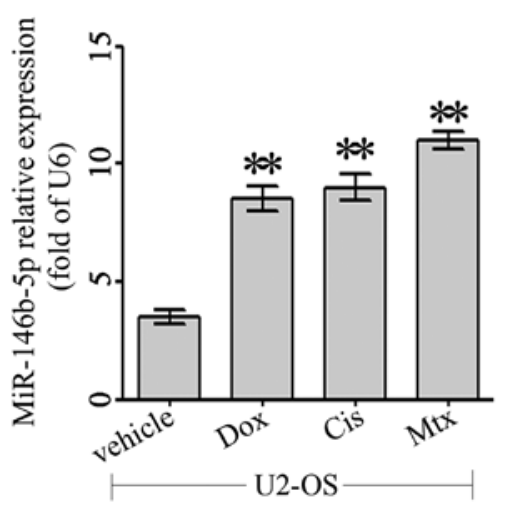

C

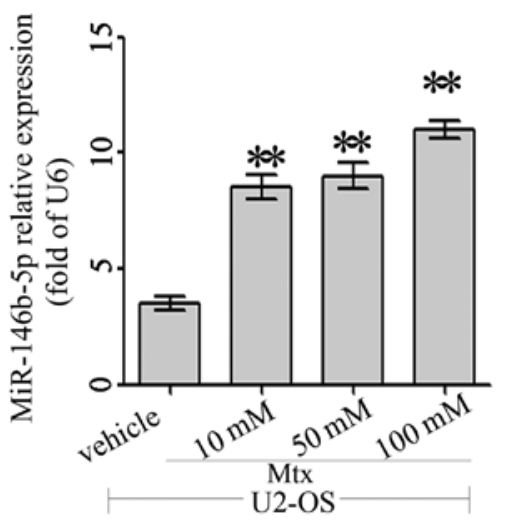

B

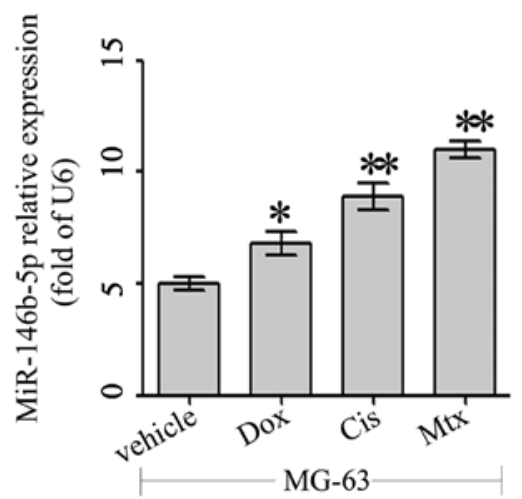

D

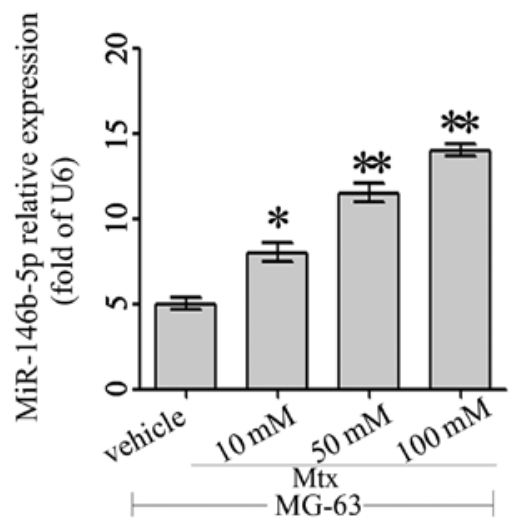

Figure 2. The expression levels of miR-146b-5p in osteosarcoma cell lines after chemotherapy. (A) U-2 OS and (B) MG-63 cell lines were treated with vehicle (normal saline), Dox $(0.2 \mathrm{mg} / \mathrm{ml})$, Cis $(20 \mathrm{mM})$, and Mtx $(50 \mathrm{mM})$ for $24 \mathrm{~h}$ and then the expression of miR-146b-5p was quantified by qRT-PCR. (C) U-2 OS and (D) MG-63 cell lines were treated with vehicle (normal saline), $5 \mathrm{mM} \mathrm{Mtx}, 50 \mathrm{mM}$ Mtx and $100 \mathrm{mM}$ Mtx for $24 \mathrm{~h}$ and then the expression of miR-146b-5p was quantified by qRT-PCR. $\left({ }^{*} \mathrm{P}<0.05,{ }^{* *} \mathrm{P}<0.01\right)$.

As compared with non-chemotherapy group, miR-146b-5p levels were prominently upregulated in chemotherapy group $(\mathrm{P}<0.05$; Fig. 1B), indicating that miR-146b-5p was involved in chemoresistence. Moreover, miR-146b-5p levels were obviously increased in tumor tissues for patients with tumor recurrence and lung metastasis as compared with those in tumor tissues arising from patients without tumor recurrence or metastasis $(\mathrm{P}<0.05, \mathrm{P}<0.05$; Fig. $1 \mathrm{C}$ and $\mathrm{D})$, suggesting that miR-146b-5p was correlated with metastasis and recurrence of osteosarcoma. In addition, we analyzed miR146b-5p expression in osteosarcoma cell lines (HOS, MG-63 and U2-OS) and human fetal osteoblastic 1.19 (hFOB1.19) cells. The miR-146b-5 expression was significantly upregulated in all osteosarcoma cell lines as compared with that in hFOB1.19 ( $\mathrm{P}<0.05$; Fig. 1D). These data indicate that elevated miR-146b-5p expression contributed to chemoresistence and metastatic potential of osteosarcoma cells.

Anticancer agents promote miR-146b-5p expression in osteosarcoma cells. The role of miR-146b-5p in different cancer cells is controversial. The exact role of miR-146b-5p in osteosarcoma remains to be clarified. To address this question, the expression of miR-146b-5p in human osteosarcoma MG-63 and U-2 OS cell lines after anticancer agents was evaluated using qRT-PCR. As doxorubicin (Dox), cisplatin (Cis), and methotrexate (Mtx) are commonly used anticancer agents in osteosarcoma, we assayed the effects of the anticancer agents on the expression of miR-146b-5p. As shown in Fig. 2A and $\mathrm{B}$, anticancer agents significantly enhanced expression of miR-146b-5p in the human osteosarcoma cell lines MG-63, and U-2 OS. Moreover, this effect was dose-dependent in the case of Mtx in MG-63 and U-2 OS cell lines (Fig. 2C and D). These findings show that miR-146b-5p expression was upregulated during chemotherapy in osteosarcoma cells.

miR-146b-5p promotes migration and invasion of osteosarcoma in vitro. Next, we applied a lentivirus system to make stable cell lines to knock down and overexpress miR-146b-5p based on the osteosarcoma cell line MG-63 including a blank group (untransfected cells), a control group (cells transfected with the control lentivirus), an OE group (overexpressing miR-146b-5p) and a KD group (knocked down miR-146b-5p). The miR-146b-5p expression levels in these groups were evaluated using qRT-PCR. As expected, the expression of miR-146b-5p was dramatically suppressed in KD group cells $(\mathrm{P}<0.05)$ while increased in the OE group $(\mathrm{P}<0.01$; Fig. 3A).

We then performed Transwell migration and invasion assay to investigate the effects of miR-146b-5p on the migratory and invasive behavior of osteosarcoma cells in vitro. In the migration assay, the number of OE MG-63 cells $(63.0 \pm 5.9$, $\mathrm{P}<0.01)$ passing through the Matrigel were significantly higher 
A

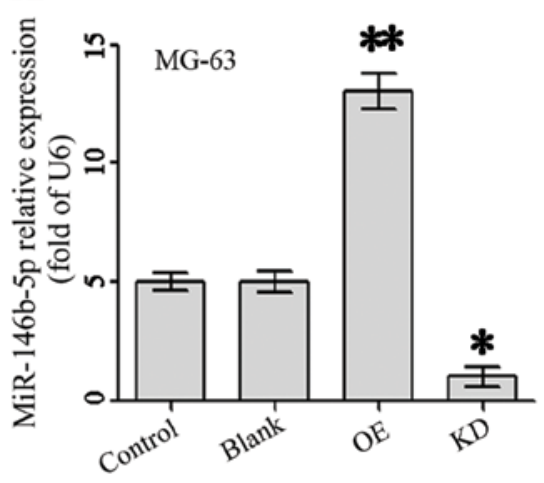

D

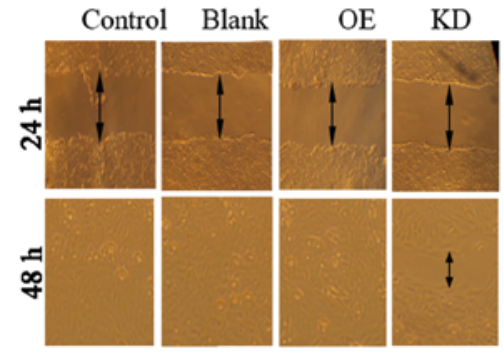

B

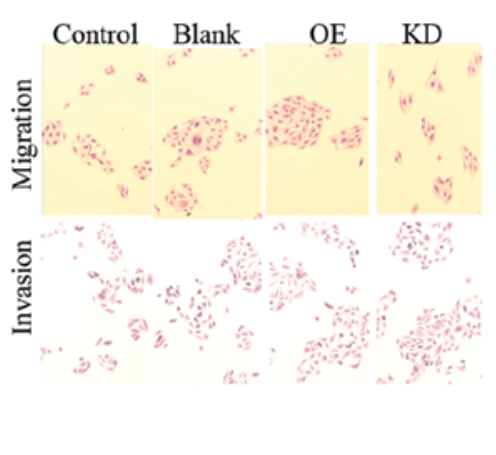

$\mathbf{E}$

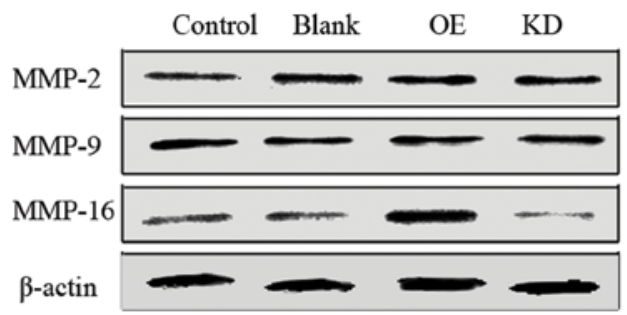

C
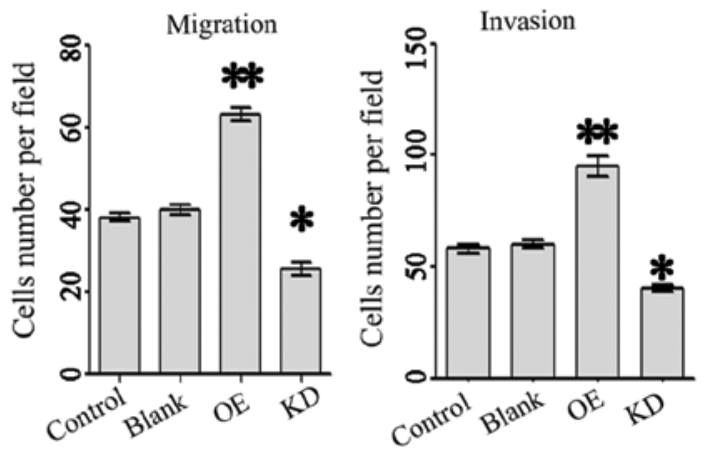

F

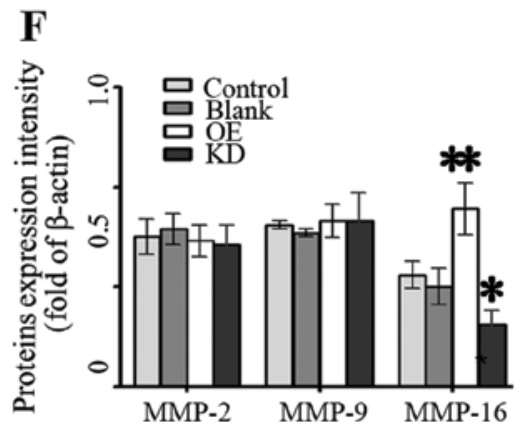

Figure 3. miR-146b-5p inhibits migration and invasion of osteosarcoma in vitro. (A) the expression of miR-146b-5p was analyzed in MG-63 cells by qRT-PCR. (B) Representative images of migrated and invaded MG-63 cells on the membrane at a magnification of x200. (C) Quantitative results for the migration and invasion ability of each group of MG-63 cells are shown as migrated and invaded cell number, $24 \mathrm{~h}$ after incubation. (D) MG-63 cells were seeded in 12 -well plates and wounds were created the next day. Images were taken at 24 and $48 \mathrm{~h}$, respectively, after the wound was made. Representative blots (E) and quantitative results $(\mathrm{F})$ of western blot analysis for MMP-2, MMP-9 and MMP-16 protein expression. The expression of MMP-16 protein was significantly increased in OE group while decreased in KD group. Yet, no difference was found in the expression of MMP- 2 and MMP-9 among the three groups. The data are presented as the means $\pm \mathrm{SD}$, the data were from three independent experiments, ${ }^{*} \mathrm{P}<0.05,{ }^{* * *} \mathrm{P}<0.01$.

than control cells $(40.0 \pm 3.8)$ (Fig. 3C). Whereas, the number of KD MG-63 cells $(25.5 \pm 6.0, \mathrm{P}<0.05)$ were significantly less than that in control cells $(40.0 \pm 3.8)$. No significant difference was observed between the blank and control cells $(\mathrm{P}=0.68)$ (Fig. 3C). Invasion assay showed that the number of $\mathrm{OE}$ cells $(95.0 \pm 15.0, \mathrm{P}<0.01)$ passing through the Matrigel were significantly higher than control cells (58.6 \pm 6.5$)$ (Fig. 3C). Similarly, KD MG-63 cells were significantly less than that in control cells $(\mathrm{P}<0.05)$ (Fig. 3D). These results strongly indicated that miR-146b-5p played a role in the migratory and invasive potential of osteosarcoma in vitro. In addition, wound healing assay was also used to examine the effects of miR-146b-5p on the migration ability. Cells in OE groups exhibited an obvious increase in migration rate as compared to the other three groups. KD MG-63 cells slightly failed to close the wound at $72 \mathrm{~h}$ after incubation, whereas the other three groups were able to close the wound at the same time-point (Fig. 3D).

In addition, western blot assay was performed to investigate the effect of miR-146b-5p on the endogenous expression of MMP-2, MMP-9 and MMP-16 protein since they were involved in tumor invasion and metastasis based on previous research (22-25). As shown in Fig. 3C, a significant increase of MMP-16 expression in the OE group while a reduction in the KD group as compared with control groups was observed $(\mathrm{P}<0.05)$. These data demonstrated that miR-146b-5p might promote osteosarcoma invasion and metastasis through regulation of MMP-16 expression.
miR-146b-5p increased chemoresistance in osteosarcoma MG-63 cells. To confirm the role of miR-146b-5p in osteosarcoma, the cell proliferation were assessed in the MG-63 cells with overexpressed or knocked down miR-146b-5p. As a result, neither the knockdown nor overexpression of miR146b-5p could alter cell growth in MG-63 cells (Fig. 4A).

Next, to explore the potential role for miR-146b-5p in chemoresistance, apoptosis in MG-63 cells was induced by vehicle (veh), doxorubicin (Dox), cisplatin (Cis) and methotrexate (Mtx). Overexpression of miR-146b-5p increased chemoresistance, while knockdown of miR-146b-5p in these cells rendered them significantly more sensitive to Dox-, Cis-, and Mtx-induced cell apoptosis (Fig. 4B). In the case of cisplatin, the apoptosis markers, cleaved caspase-3 and cleaved PARP proteins were assessed in $\mathrm{KD}, \mathrm{OE}$ and control group, cleaved caspase- 3 and cleaved PARP proteins were suppressed in OE group. However, cleaved caspase- 3 and cleaved PARP were increased in KD group (Fig. 4C and D). These data suggested that miR-146b-5p increased chemoresistance possibly through suppressing the apoptotic pathway.

We investigated whether the Wnt/ $\beta$-catenin signaling was involved in the miR-146b-5p mediated chemoresistance as previous studies demonstrated that $\mathrm{Wnt} / \beta$-catenin played an important role in chemoresistance $(26,27)$. The cell apoptosis was assessed in osteosarcoma overexpressing miR-146b-5p (OE group) in combination with or without the Wnt inhibitor (IWR-1-endo). Our results showed that the decrease in apop- 
A

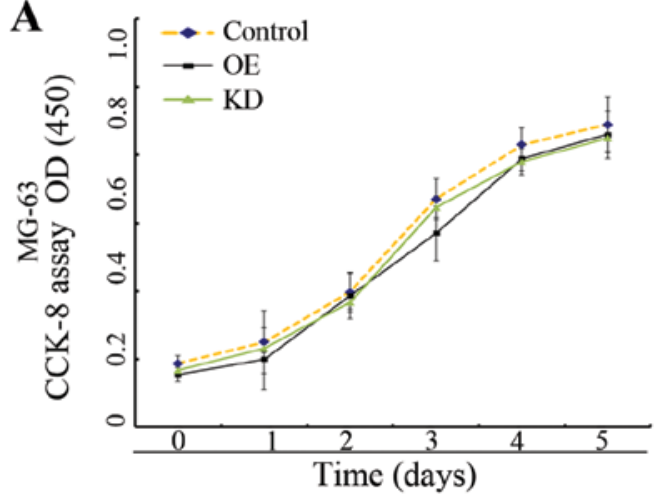

C

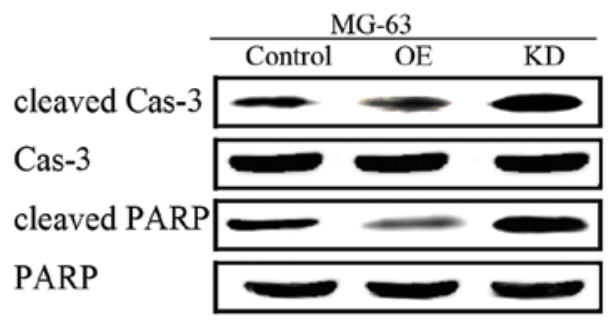

$\mathbf{E}$

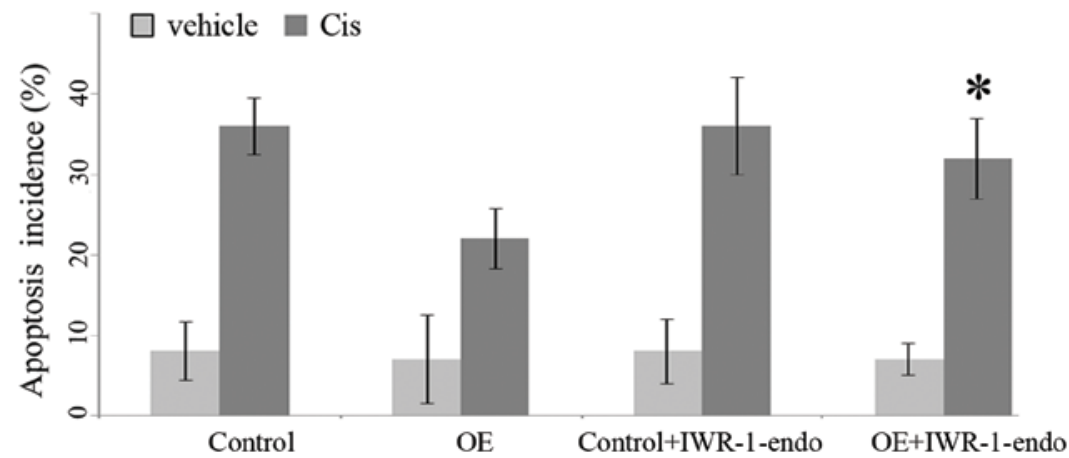

B

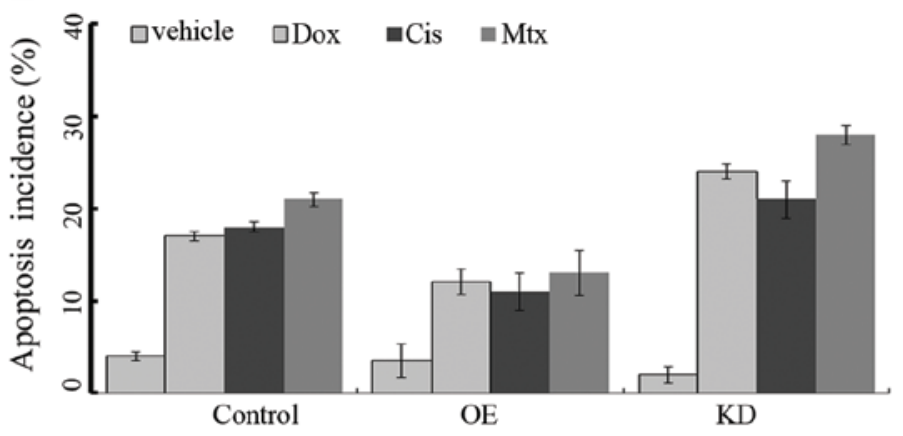

D

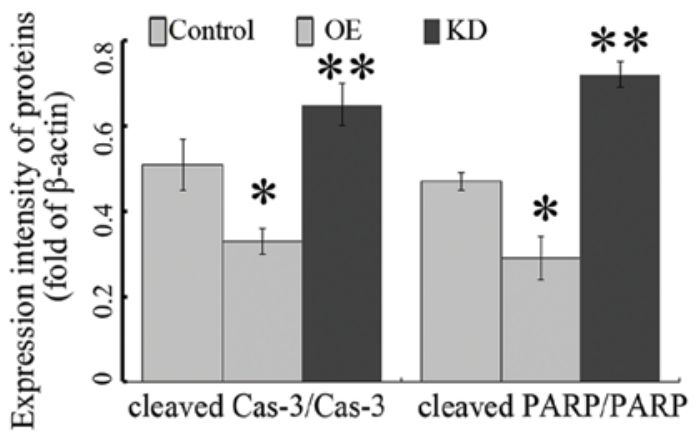

Figure 4. Overexpression of miR-146b-5p increases resistance to chemotherapy in vitro. (A) CCK-8 assay for miR-146b-5p overexpression (OE) knockdown (KD) and control. No difference was found among OE, KD and control groups. (B) Annexin V-FITC/PI staining for apoptotic cells. MG63 cells in OE, KD and control groups were treated with vehicle (normal saline), Dox $(0.2 \mathrm{mg} / \mathrm{ml})$, Cis $(20 \mathrm{mmol} / \mathrm{l})$, and Mtx $(50 \mathrm{mmol} / \mathrm{l})$ for $24 \mathrm{~h}$ and then the apoptosis incidence was quantified by flow cytometer. Overexpression of miR-146b-5p reduced the incidence of apoptosis induced by Dox, Cis and Mtx. ( $\mathrm{P}<0.05$, ${ }^{* *} \mathrm{P}<0.01 \mathrm{vs}$. vehicle group). Representative blots (C) and quantitative results (D) of western blot analysis for cleaved caspase-3, caspase-3, cleaved PARP, and PARP protein expression. Both the ratio of cleaved caspase-3 to caspase-3 expression and the ratio of cleaved PARP to PARP were significantly reduced in OE group compared with KD and control groups. (E) Annexin V-FITC/PI staining for apoptotic cells. MG63 cells in OE and control groups were treated with vehicle (normal saline) or Cis $(20 \mathrm{mmol} / \mathrm{l})$ in combination with $300 \mathrm{nM}$ IWR-1-endo. The apoptosis was assessed by flow cytometer. * $\mathrm{P}<0.05 \mathrm{OE}+\mathrm{IWR}-1$-endo vs. OE.

tosis of OE cells caused by miR-146b-5p overexpression was significantly suppressed by IWR-1-endo (Fig. 4E). These results suggested that the effect of miR-146b-5p on increasing the chemoresistance of osteosarcoma cells was mediated by Wnt $/ \beta$-catenin signaling.

ZNRF3 is a target of miR-146b-5p, and highly expressed in osteosarcoma after chemotherapy in vivo and in vitro. To investigate the mechanism of miR-146b-5p in chemoresistance, bioinformatics analysis demonstrated that the 3'-UTR region of ZNRF3 were identified as the binding sites for miR-146b-5p (Fig. 5A). To further verify that ZNRF3 was a direct target of miR-146b-5p, luciferase assays were performed and mimic NC, miR-146b-5p mimics, anti-NC or anti-miR-146b-5p were transfected into the MG-63 cell lines. Our results demonstrated that miR-146b-5p expression was high in miR-146b-5p mimics group (Fig. 5B). Luciferase reporter assays showed that miR-146b-5p mimics significantly inhibited the luciferase activity of ZNRF3 3'-UTR by $\sim 30 \%$ in MG-63 cells relative to the control, whereas co-transfection with anti-miR-146b-5p significantly promoted luciferase activity by $160 \%(\mathrm{P}<0.05)$ (Fig. 5C).

To further confirm that miR-146b-5p modulates the expression of ZNRF3, MG-63 and U-2 OS cells were transfected with miR-146b-5p mimics, anti-miR-146b mimics or the respective controls. Subsequently, the protein expression of ZNRF3 was analyzed by western blot analysis. Transfection of the miR-146b-5p mimics significantly decreased the 
A

$\begin{array}{ll}\text { Target Region(poorly conserved) } & \text { 5' CGAGGUGUGGGAUUGAGUUCUCU } \\ \text { Position 288-294 of ZNRF3 3' UTR } & \text { 3' UCGGAUACCUUAAGUCAAGAGU } \\ \text { Hsa-miR-146b-5p } & \text { 5' CAUUUAUGGAAAUAUGUUCUCAG } \\ \text { Position 3620-3626 of ZNRF3 3' UTR } & \text { 3' UCGGAUACCUUAAGUCA'AGAGU } \\ \text { Hsa-miR-146b-5p }\end{array}$

B

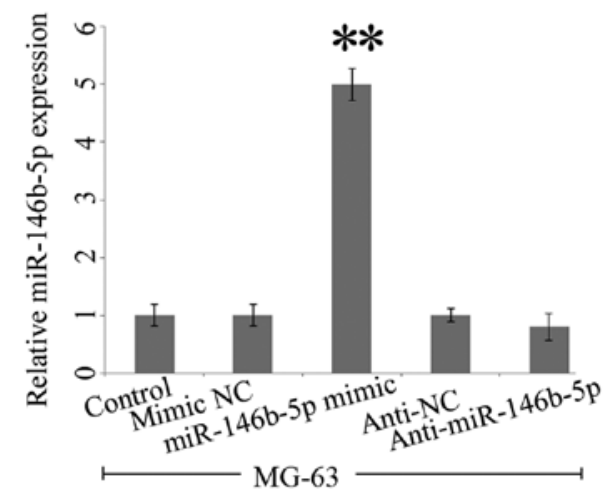

D
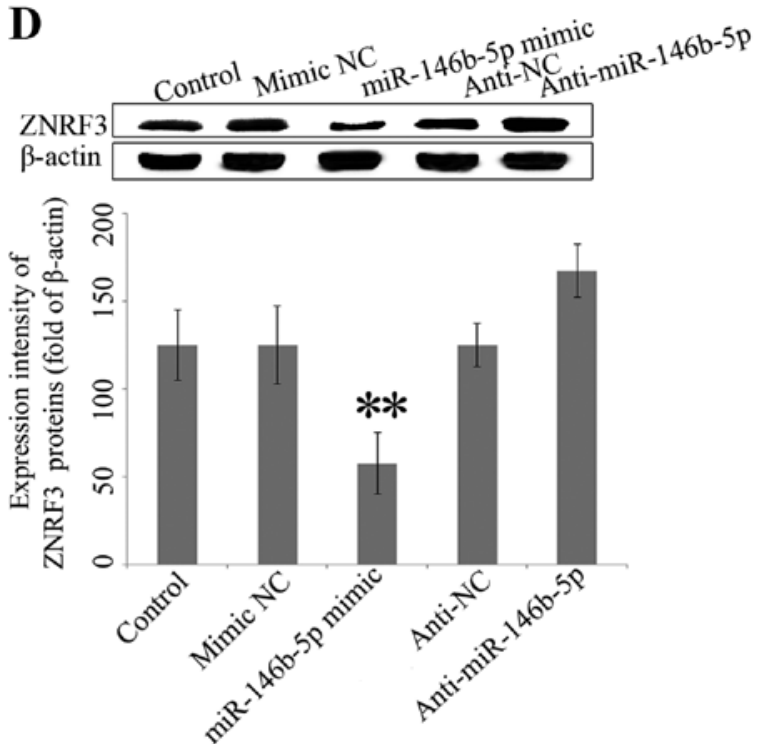

C

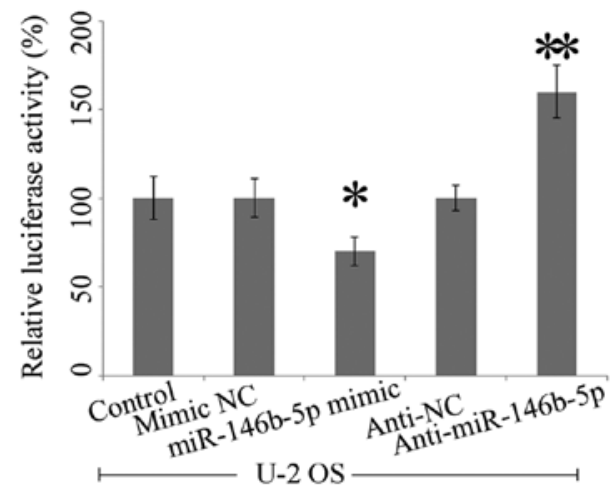

$\mathbf{E}$
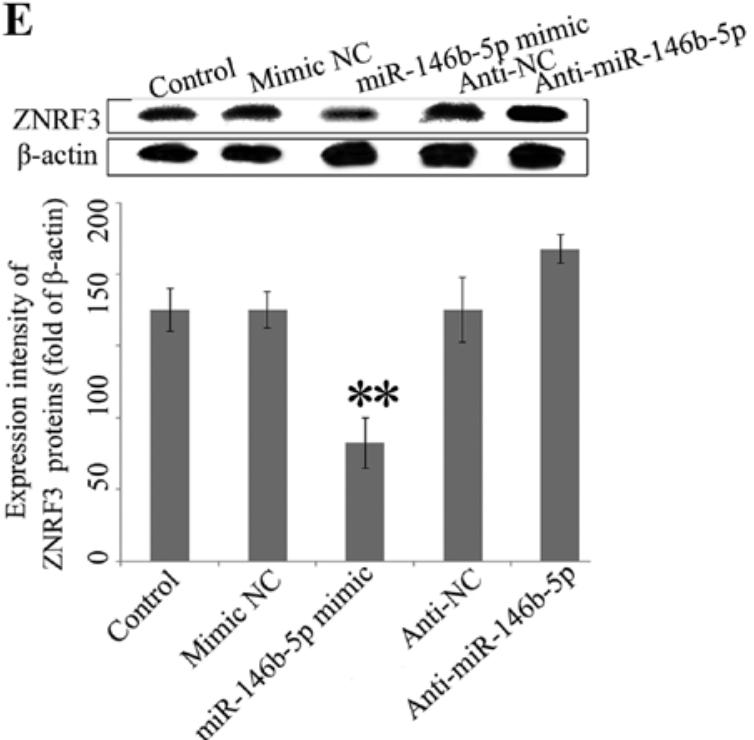

Figure 5. ZNRF3 is a target of miR-146b-5p in osteosarcoma. (A) The predicted binding site of miR-146b-5p on 3'UTR ZNRF3. (B) MG-63 cells were transfected or not with mimic NC, miR-146b-5p mimic, anti-NC or anti-miR-146b, and miR-146b-5p expression was analyzed in MG-63 cells by real-time PCR. (C) A luciferase reporter plasmid carrying ZNRF3 3'UTR was transfected into MG-63 cells, co-transfected with mimic NC or miR-146b-5p mimic or with anti-NC or anti-miR-146b-5p and luciferase activity was measured. (D) MG-63 and (E) U-2 OS cells were transfected or not with mimic NC, miR-146b-5p mimic, anti-NC or anti-miR-146b and ZNRF3 protein expression was analyzed by western blot analysis. ${ }^{*} \mathrm{P}<0.05,{ }^{* *} \mathrm{P}<0.01, \mathrm{vs}$. control.

protein level of ZNRF3, whereas anti-miR-146b-5p had the opposite effects (Fig. 5D and E).

\section{Discussion}

Osteosarcoma is one of the predominant tumors in children and chemoresistance is a leading cause of mortality for osteosarcoma patients. The molecular mechanism for chemoresistance remains unclear. Previously, we identified several genes and proteins regarding chemoresistance ability, including, high mobility group nucleosome binding domain $5(20,28)$, tumor necrosis factor- $\alpha$-inducible protein-1 (29) and nucleosome-binding protein (30). However, knockdown of these genes only partly suppress the chemoresistance, suggesting that these genes exert only a part of their biological effects as chemoresistance. MicroRNAs are important regulators in tumorigenesis as well as chemoresistance. Increasing evidence demonstrated that miR-146b-5p played an oncogenic role in papillary thyroid carcinoma (31), pancreatic cancer (21) and lung cancer cells (32). However, to date, the role of miR-146b-5p in osteosarcoma is unclear. 
To confirm the biological role of miR-146b-5p in osteosarcoma, we first assessed the miR-146b-5p expression in human osteosarcoma tissues before and after chemotherapy, tumor and ANCT, tumor with metastasis or without. The expression of miR-146b-5p was also evaluated in hFOB1.19 osteoblast cell line, HOS, MG-63 and U2-OS osteosarcoma cell lines. As expected, these results suggested that miR-146b-5p was highly expressed in osteosarcoma tissues, especially after chemotherapy. Furthermore, the expression of miR-146b-5p was upregulated during chemotherapy in osteosarcoma cells in vitro. These data suggested that miR-146b-5p was possibly involved in chemoresistence and metastatic potential of osteosarcoma cells. To confirm our hypothesis, we applied lentivirus system to make stable cell lines to knock down and overexpress miR-146b-5p and our results showed that miR-146b-5p promoted osteosarcoma invasion and metastasis through regulation of MMP-16 expression. In the present study, gain-of-function studies demonstrated that miR-146b-5p overexpression reduced the apoptosis incidence and apoptosis marker expression, suggesting at miR-146b-5p plays a crucial role in the chemoresistance of osteosarcoma.

The Wnt/ $\beta$-catenin pathway plays a crucial role in the chemoresistance of osteosarcoma (33) and we also investigated whether Wnt/ $\beta$-catenin pathway was involved in chemoresistance of osteosarcoma. As expected, IWR-1-endo, an inhibitor of Wnt/ $\beta$-catenin signaling, could restore the decrease in apoptosis of OE cells caused by miR-146b-5p overexpression, suggested that the effect of miR-146b-5p on increasing the chemoresistance is mediated by $\mathrm{Wnt} / \beta$-catenin signaling.

To investigate the mechanism of miR-146b-5p in regulating Wnt/ $\beta$-catenin signaling, Bioinformatics analysis was performed which showed that the 3'-UTR region of ZNRF3 were the binding sites for miR-146b-5p. A similar role for ZNRF3 was demonstrated in human gastric adenocarcinoma and papillary thyroid carcinoma, showing that ZNRF3 promoted apoptosis through the modulation of the $\mathrm{Wnt} / \beta$ catenin signaling pathway $(13,34)$.

To the best of our knowledge, this is the first report of miR-146b-5p in the chemoresistance of osteosarcoma. Although our research demonstrated that miR-146b-5p participated in migration, invasion and chemoresistance in osteosarcoma, the use of osteosarcoma cells from two cell lines provide very limited evidence. Further research using more cell lines and primary tumors in animal studies is necessary to confirm the findings of the present study. In conclusion, our investigation revealed that the miR-146b-5p affects migration, invasion and chemoresistance in osteosarcoma possibly via downregulation of ZNRF3.

\section{References}

1. Jemal A, Bray F, Center MM, Ferlay J, Ward E and Forman D: Global cancer statistics. CA Cancer J Clin 61: 69-90, 2011.

2. Scotlandi K, Picci P and Kovar H: Targeted therapies in bone sarcomas. Curr Cancer Drug Targets 9: 843-853, 2009.

3. Lønning PE and Knappskog S: Mapping genetic alterations causing chemoresistance in cancer: Identifying the roads by tracking the drivers. Oncogene 32: 5315-5330, 2013.

4. Derdak Z, Mark NM, Beldi G, Robson SC, Wands JR and Baffy G: The mitochondrial uncoupling protein-2 promotes chemoresistance in cancer cells. Cancer Res 68: 2813-2819, 2008.
5. Yamamoto S, Tsuda H, Honda K, Onozato K, Takano M, Tamai S, Imoto I, Inazawa J, Yamada T and Matsubara O: Actinin-4 gene amplification in ovarian cancer: A candidate oncogene associated with poor patient prognosis and tumor chemoresistance. Mod Pathol 22: 499-507, 2009.

6. Zhang W, Li Q, Song C and Lao L: Knockdown of autophagyrelated protein 6 , Beclin-1, decreases cell growth, invasion, and metastasis and has a positive effect on chemotherapy-induced cytotoxicity in osteosarcoma cells. Tumour Biol 36: 2531-2539, 2014.

7. Zhang Y, Duan G and Feng S: MicroRNA-301a modulates doxorubicin resistance in osteosarcoma cells by targeting AMP-activated protein kinase alpha 1. Biochem Biophys Res Commun 459: 367-373, 2015.

8. Zhao Z, Zhang L, Yao Q and Tao Z: miR-15b regulates cisplatin resistance and metastasis by targeting PEBP4 in human lung adenocarcinoma cells. Cancer Gene Ther 22: 108-114, 2015.

9. Zhao Y, Zhao L, Ischenko I, Bao Q, Schwarz B, Nieß H, Wang Y, Renner A, Mysliwietz J, Jauch KW, et al: Antisense inhibition of microRNA-21 and microRNA-221 in tumor-initiating stem-like cells modulates tumorigenesis, metastasis, and chemotherapy resistance in pancreatic cancer. Target Oncol: Feb 3, 2015. (Epub ahead of print).

10. Lian F, Cui Y, Zhou C, Gao K and Wu L: Identification of a plasma four-microRNA panel as potential noninvasive biomarker for osteosarcoma. PLoS One 10: e0121499, 2015.

11. Luo XJ, Tang DG, Gao TL, Zhang YL, Wang M, Quan ZX and Chen J: MicroRNA-212 inhibits osteosarcoma cells proliferation and invasion by down-regulation of Sox4. Cell Physiol Biochem 34: 2180-2188, 2014.

12. Nata T, Fujiya M, Ueno N, Moriichi K, Konishi H, Tanabe H, Ohtake T, Ikuta K and Kohgo Y: MicroRNA-146b improves intestinal injury in mouse colitis by activating nuclear factor- $\kappa \mathrm{B}$ and improving epithelial barrier function. J Gene Med 15: 249-260, 2013.

13. Deng X, Wu B, Xiao K, Kang J, Xie J, Zhang X and Fan Y: MiR-146b-5p promotes metastasis and induces epithelialmesenchymal transition in thyroid cancer by targeting ZNRF3. Cell Physiol Biochem 35: 71-82, 2015.

14. Wu PY, Zhang XD, Zhu J, Guo XY and Wang JF: Low expression of microRNA-146b-5p and microRNA-320d predicts poor outcome of large B-cell lymphoma treated with cyclophosphamide, doxorubicin, vincristine, and prednisone. Hum Pathol 45: 1664-1673, 2014

15. Dieudonné FX, Marion A, Marie PJ and Modrowski D: Targeted inhibition of T-cell factor activity promotes syndecan-2 expression and sensitization to doxorubicin in osteosarcoma cells and bone tumors in mice. J Bone Miner Res 27: 2118-2129, 2012.

16. Kreslova J, Machon O, Ruzickova J, Lachova J, Wawrousek EF, Kemler R, Krauss S, Piatigorsky J and Kozmik Z: Abnormal lens morphogenesis and ectopic lens formation in the absence of betacatenin function. Genesis 45: 157-168, 2007.

17. Machon O, Kreslova J, Ruzickova J, Vacik T, Klimova L, Fujimura N, Lachova J and Kozmik Z: Lens morphogenesis is dependent on Pax6-mediated inhibition of the canonical Wnt/ beta-catenin signaling in the lens surface ectoderm. Genesis 48: 86-95, 2010.

18. Jiang X, Hao HX, Growney JD, Woolfenden S, Bottiglio C, Ng N, Lu B, Hsieh MH, Bagdasarian L, Meyer R, et al: Inactivating mutations of RNF43 confer Wnt dependency in pancreatic ductal adenocarcinoma. Proc Natl Acad Sci USA 110: 12649-12654, 2013.

19. Ryland GL, Hunter SM, Doyle MA, Rowley SM, Christie M, Allan PE, Bowtell DD, Gorringe KL and Campbell IG; Australian Ovarian Cancer Study Group: RNF43 is a tumour suppressor gene mutated in mucinous tumours of the ovary. J Pathol 229: 469-476, 2013.

20. Zhou X, Yuan B, Yuan W, Wang C, Gao R and Wang J: The expression and clinical significance of high mobility group nucleosome binding domain 5 in human osteosarcoma. Tumour Biol 35: 6539-6547, 2014.

21. Lin F, Wang X, Jie Z, Hong X, Li X, Wang M and Yu Y: Inhibitory effects of miR-146b-5p on cell migration and invasion of pancreatic cancer by targeting MMP16. J Huazhong Univ Sci Technolog Med Sci 31: 509-514, 2011

22. Zhao Z, Tao L, Shen C, Liu B, Yang Z and Tao H: Silencing of Barkor/ATG14 sensitizes osteosarcoma cells to cisplatin-induced apoptosis. Int J Mol Med 33: 271-276, 2014. 
23. Wang J, Shi Q, Yuan TX, Song QL, Zhang Y, Wei Q, Zhou L, Luo J,Zuo G, Tang M, et al: Matrix metalloproteinase 9 (MMP-9) in osteosarcoma: Review and meta-analysis. Clin Chim Acta 433: 225-231, 2014.

24. Shang HS, Chang JB, Lin JH, Lin JP, Hsu SC, Liu CM, Liu JY, Wu PP, Lu HF, Au MK, et al: Deguelin inhibits the migration and invasion of U-2 OS human osteosarcoma cells via the inhibition of matrix metalloproteinase-2/-9 in vitro. Molecules 19: 16588-16608, 2014.

25. Li Y, Wang Y, Yu L, Sun C, Cheng D, Yu S, Wang Q, Yan Y, Kang C, Jin S, et al: miR-146b-5p inhibits glioma migration and invasion by targeting MMP16. Cancer Lett 339: 260-269, 2013.

26. Aguilera Ó, González-Sancho JM, Zazo S, Rincón R, Fernández AF, Tapia O, Canals F, Morte B, Calvanese V, Orgaz JL, et al: Nuclear DICKKOPF-1 as a biomarker of chemoresistance and poor clinical outcome in colorectal cancer. Oncotarget 6: 5903-5917, 2015.

27. Scholten DJ II, Timmer CM, Peacock JD, Pelle DW, Williams BO and Steensma MR: Down regulation of Wnt signaling mitigates hypoxia-induced chemoresistance in human osteosarcoma cells. PLoS One 9: e111431, 2014.

28. Yang C, Gao R, Wang J, Yuan W, Wang C and Zhou X: Highmobility group nucleosome-binding domain 5 increases drug resistance in osteosarcoma through upregulating autophagy. Tumour Biol 35: 6357-6363, 2014
29. Zhang CL, Wang C, Yan WJ, Gao R, Li YH and Zhou XH: Knockdown of TNFAIP1 inhibits growth and induces apoptosis in osteosarcoma cells through inhibition of the nuclear factor- $\kappa \mathrm{B}$ pathway. Oncol Rep 32: 1149-1155, 2014.

30. Liang G, Xu E, Yang C, Zhang C, Sheng X and Zhou X: Nucleosome-binding protein HMGN2 exhibits antitumor activity in human $\mathrm{SaO} 2$ and U2-OS osteosarcoma cell lines. Oncol Rep 33: 1300-1306, 2015

31. He H, Jazdzewski K, Li W, Liyanarachchi S, Nagy R, Volinia S, Calin GA, Liu CG, Franssila K, Suster S, et al: The role of microRNA genes in papillary thyroid carcinoma. Proc Natl Acad Sci USA 102: 19075-19080, 2005.

32. Patnaik SK, Kannisto E, Mallick $R$ and Yendamuri $S$ : Overexpression of the lung cancer-prognostic miR-146b microRNAs has a minimal and negative effect on the malignant phenotype of A549 lung cancer cells. PLoS One 6: e22379, 2011.

33. Lin $\mathrm{CH}$, Ji T, Chen $\mathrm{CF}$ and Hoang $\mathrm{BH}$ : Wnt signaling in osteosarcoma. Adv Exp Med Biol 804: 33-45, 2014.

34. Zhou Y, Lan J, Wang W, Shi Q, Lan Y, Cheng Z and Guan H: ZNRF3 acts as a tumour suppressor by the Wnt signalling pathway in human gastric adenocarcinoma. J Mol Histol 44: 555-563, 2013. 